16

\title{
Рассеяние оптического излучения шероховатой разномасштабной зеркальной поверхностью
}

\author{
(C) П.А. Головинский, Д.К. Проскурин \\ Воронежский государственный технический университет, \\ 394006 Воронеж, Россия \\ e-mail: golovinski@bk.ru
}

Поступила в редакцию 04.02.2019 г.

В окончательной редакции 12.09.2019 г.

Принята к публикации 15.11.2019 г.

В приближении физической оптики рассмотрено обратное рассеяние световых волн шероховатой поверхностью с одномерным рельефом из разномасштабных зеркальных фрагментов, действующей как фазовый экран. Получены асимптотические угловые распределения в приближении диффузии фазы, а с целью учета сложной статистики неровностей применен численный метод фазового скачка. Для периодически модулированной шероховатой поверхности результаты численных расчетов методом фазового скачка и методом касательных отражающих плоскостей сопоставлены с экспериментальными данными. Представлены угловые зависимости обратного рассеяния, демонстрирующие влияние распределения Релея шероховатостей на интенсивность рассеяния, и показано сужение области углов обратного рассеяния под влиянием дополнительного крупномасштабного случайного профилирования поверхности.

Ключевые слова: ступенчатая поверхность, двухмасштабная модель, дифракция Фраунгофера, диффузия фазы.

DOI: $10.21883 /$ OS.2020.03.49068.42-19

\section{Введение}

В классической электродинамике, оптике и акустике границы раздела сред обычно считаются гладкими, в то время как реальные объекты имеют в той или иной степени неровную поверхность [1]. В силу этого рассеяние волн шероховатыми поверхностями является важным вопросом в различных областях науки и техники, связанных с измерениями, включая радиотехнические и лазерные системы локации [2-4], а также метрологию [5]. Целью теоретического описания рассеяния волн шероховатыми поверхностями является построение моделей, позволяющих предсказать структуру рассеянного поля по характеристикам поверхности или решить обратную задачу определения этих характеристик по измерению параметров рассеянного излучения [6].

При решении данного круга задач приближенные модели являются необходимым инструментом ввиду чрезвычайной сложности прямых численных расчетов, требуемых для описания реальных проблем рассеяния. Это определило развитие совокупности моделей, которые для электромагнитного излучения более или менее последовательно выводятся из уравнений Максвелла [7-9]. Одним из наиболее популярных приближений остается метод возмущений, основанный на разложении амплитуды рассеяния волны по малому параметру отношения высоты пологих неровностей к длине волны, который был впервые введен Релеем [10]. Другой ключевой подход состоит в использовании приближения Кирхгофа в сочетании с приближением касательных отражающих плоскостей, областью применимости которого является описание рассеяния на крупных и плавных неоднородностях с радиусом кривизны, существенно превышающим длину волны $[11,12]$. Более совершенные модели основываются на интегральных уравнениях и учитывают многократное рассеяние [12-14], однако они значительно сложнее в вычислительном отношении, особенно в коротковолновой области.

Основная проблема, которая выявляется при сравнении различных приближений, состоит в отсутствии равномерного по длине волны аналитического метода расчета, работающего с равным успехом как в области коротких, так и в области длинных волн, и трудности в описании рассеяния, когда характерные размеры неоднородностей становятся порядка длины волны. Еще одним ограничением теории рассеяния волн шероховатыми поверхностями является отсутствие сложных комбинированных моделей поверхностей, в том числе аппликативных поверхностей, состоящих из чередующихся участков с различными характеристиками шероховатости.

Наличие двух характерных областей в угловой зависимости интенсивности света, отраженного сложной случайной шероховатой поверхностью, воспроизводится в двухмасштабной модели [15-22], но в ней разные масштабы четко разграничены, а рассеяние в пределах каждого масштаба неоднородности описывается отдельно методом малых возмущений Релея или в приближении Кирхгофа $[23,24]$. Отметим, что двухмасштабная модель применялась преимущественно к решению задач рассеяния волн шероховатыми поверхностями с гауссовой статистикой неровностей. Для рассмотрения бо- 
лее сложных статистик мы воспользуемся специальной фазово-модулирующей поверхностью ступенчатой формы, которая позволяет существенно упростить описание рассеяния при наличии разномасштабных неоднородностей. Такая поверхность состоит из длинных идеально отражающих (зеркальных) горизонтальных параллельных полос разной ширины и высоты над подстилающей плоской поверхностью. Таким образом, высота рельефа поверхности зависит только от одной координаты, и модель является одномерной. За счет изменения высоты отражающих элементов над подстилающей поверхностью происходит модуляция фазы отраженной волны, на которую при больших расстояниях от рассеивающей поверхности накладываются изменения, вызванные разной дистанцией распространения волны от текущей точки экрана до точки наблюдения. Такой подход к описанию рассеяния совпадает с методом локального фазового скачка $[25,26]$. Аналогичная модель использована в работе [27], где рассмотрена случайная по высоте мозаика поверхности из одинаковых отражающих квадратных элементов. Фактически обсуждаемая модель представляет собой вариант приближения Рытова [6,7] для отражения, в котором эффект модуляции волны неровной поверхностью сводится к воздействию на нее случайного фазового экрана. В настоящей работе рассмотрена задача обратного рассеяния поверхностью со сложной статистикой шероховатостей как наиболее интересного для локации.

\section{Уравнения обратного рассеяния зеркальной ступенчатой поверхностью}

В теоретическом описании рассеяния волн на шероховатых поверхностях ключевым является интегральное уравнение Кирхгофа-Гельмгольца [12]. Мы ограничимся скалярной задачей рассеяния монохроматической волны с временной зависимостью $\exp (-i \omega t)$. Если предположить поле вблизи поверхности известным, то для поля рассеянной волны $u^{\prime}\left(\mathbf{R}_{1}\right)$ в точке наблюдения $\mathbf{R}_{1}$ справедлива формула

$$
u^{\prime}\left(\mathbf{R}_{1}\right)=\int_{S}\left(u(\mathbf{R}) \frac{\partial G\left(\mathbf{R}_{1}, \mathbf{R}\right)}{\partial n}-G\left(\mathbf{R}_{1}, \mathbf{R}\right) \frac{\partial u(\mathbf{R})}{\partial n}\right) d S
$$

где поле $u(\mathbf{R})$ вблизи поверхности интегрирования $S$ и производная по направлению нормали $\frac{\partial u(\mathbf{R})}{\partial n}$ взяты с наружной стороны поверхности. Функция Грина $G\left(\mathbf{R}_{1}, \mathbf{R}\right)$ в области свободного пространства имеет вид

$$
G\left(\mathbf{R}_{1}, \mathbf{R}\right)=\frac{\exp \left(i k\left|\mathbf{R}_{1}-\mathbf{R}\right|\right)}{4 \pi\left|\mathbf{R}_{1}-\mathbf{R}\right|}
$$

Формально уравнение (1) является точным, а проблема нахождения рассеянного излучения сводится к необходимости определить поле $u(\mathbf{R})$ вблизи поверхности, для чего существуют различные приближенные методы.
Для полностью отражающих поверхностей имеются два варианта граничных условий $[28,29]$ : условие Неймана, когда нормальная производная равна нулю, т. е.

$$
\frac{\partial u(\mathbf{R})}{\partial n}=0, \quad \mathbf{R} \in S,
$$

(акустически жесткое тело), и условие Дирихле

$$
u(\mathbf{R})=0, \quad \mathbf{R} \in S,
$$

(акустически мягкое тело). В приближении физической оптики (ФО) [28,30] поле на плоской поверхности рассеяния можно выразить через поле падающей волны $u^{\text {inc }}(\mathbf{R})$. На акустически мягкой поверхности полное поле равно нулю, а его нормальная производная

$$
\frac{\partial u}{\partial n}=2 \frac{\partial u^{\text {inc }}}{\partial n} .
$$

На акустически жесткой поверхности производная полного поля равна нулю, а само поле

$$
u=2 u^{\text {inc }} \text {. }
$$

Полагая, что приведенные соотношения верны для каждого локально плоского участка отражающей шероховатой поверхности, в приближении ФО получим интегральное представление для рассеянного поля $u_{s}, u_{h}$ на акустически мягкой и акустически жесткой поверхности:

$$
\begin{aligned}
& u_{S}\left(\mathbf{R}_{1}\right)=-2 \int_{S} \frac{\partial u^{\text {inc }}}{\partial n} G\left(\mathbf{R}_{1}, \mathbf{R}\right) d S, \\
& u_{h}\left(\mathbf{R}_{1}\right)=2 \int_{S} u^{\text {inc }} \frac{\partial}{\partial n} G\left(\mathbf{R}_{1}, \mathbf{R}\right) d S .
\end{aligned}
$$

Несмотря на формально разный вид, в приближении ФО обратное рассеяние от акустически мягких и жестких тел одинаковой формы и одинакового размера отличается только знаком [28]. Между акустическими и электромагнитными волнами при обратном рассеянии в приближении ФО существует соотношение эквивалентности. Рассеяние электрического поля идеально проводящей поверхностью описывается как рассеяние акустических волн на абсолютно мягкой поверхности, а рассеяние магнитного поля идеально проводящей поверхностью описывается как рассеяние акустических волн на абсолютно жесткой поверхности. Эквивалентность уравнений (2) и (3) в рассматриваемой задаче позволяет использовать далее любую из этих форм и считать задачу скалярной.

В нашей модели поверхность представляет собой совокупность зеркальных ступенек, а геометрия задачи рассеяния на поверхности одной ступеньки показана на рис. 1. Ось координат $z$ выбрана по нормали к площадке (полосе), а горизонтальные координаты $x, y$ вдоль поверхности задают двумерный вектор $\mathbf{r}=\{x, y\}$. Неровности описываются ступенчатой функцией $\xi(x)$ отклонения высоты полосы от уровня горизонтальной 


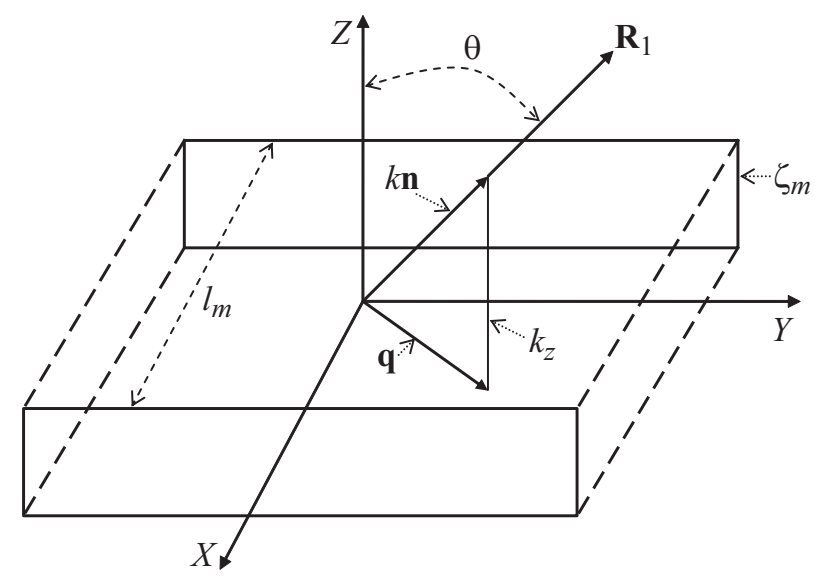

Рис. 1. Геометрия рассеяния волны отдельной ступенькой шириной $l_{m}$, высотой $\xi_{m}$ и используемые обозначения.

подстилающей поверхности. На больших расстояниях источника и приемника излучения от поверхности отражающего объекта падающую волну можно считать плоской, а условия рассеяния, соответствующими дифракции Фраунгофера. Поле плоской падающей волны с единичной амплитудой $\exp \left[i k\left(\mathbf{n}_{i}^{\perp} \mathbf{r}+z n_{i}^{2}\right)\right]$ непосредственно вблизи отражающей поверхности можно записать в виде

$$
u(\mathbf{r})=\exp \left(i k \mathbf{n}_{i}^{\perp} \mathbf{r}\right) \exp \left[-k_{i}^{2} \xi(x)\right]
$$

Здесь $k$ - волновое число, единичный вектор направления падающей волны $\mathbf{n}_{i}=\left\{\mathbf{n}_{i}^{\perp}, n_{i}^{2}\right\}$ представляется в виде совокупности составляющей $\mathbf{n}_{i}^{\perp}$ параллельной плоскости рассеивающей поверхности и вертикальной компоненты $n_{i}^{z}, k_{i}^{z}=k n_{i}^{2}$, а $k \mathbf{n}_{i}^{\perp}=\mathbf{q}_{i}-$ составляющая волнового вектора падающей волны вдоль поверхности. Уравнение (3) для рассеянной волны в дальней зоне принимает вид интеграла по ступенчатой поверхности

$$
\begin{aligned}
u_{h}\left(\mathbf{R}_{1}\right)= & -\frac{i k_{z}}{2 \pi} \int \exp \left[i \mathbf{q}_{i} \mathbf{r}-i k_{i}^{z} \zeta(x)\right] \\
& \times \frac{\exp \left[i k R_{1}-i \mathbf{q} \mathbf{r}-i k_{z} \zeta(x)\right]}{R_{1}} d \mathbf{r} .
\end{aligned}
$$

Волновой вектор рассеянной волны $\mathbf{k}=\left\{\mathbf{q}, k_{z}\right\}=$ $=k\left(\mathbf{R}_{1} / R_{1}\right)=k \mathbf{n}$, где $\mathbf{n}-$ единичный вектор направления рассеяния, а $\mathbf{q}=k \mathbf{r}_{1} / R_{1}-$ составляющая вектора $\mathbf{k}$ вдоль отражающей поверхности. Если исходить из уравнения (2), множитель $k_{z}$ перед интегралом в уравнении (4) нужно заменить на $k_{i}^{z}$. В условиях обратного рассеяния $k_{i}^{z}=-k_{z}$, в соответствии с общим утверждением об эквивалентности представлений (2) и (3), сделанным выше. При вертикальном смещении отражающей поверхности на величину $\xi(x)$ фаза отраженной волны изменяется на величину $-k_{i}^{z}(x)$. За счет изменения расстояния до точки наблюдения фаза меняется еще на $-k_{z} \xi(x)$. Обозначим поперечную компоненту вектора рассеяния $\mathbf{Q}=\mathbf{q}_{i}-\mathbf{q}$, а его продольную компоненту $K=k_{i}^{z}+k_{z}$. Для обратного рассеяния
$Q=2 k \sin \theta, K=2 k \cos \theta$, где $\theta-$ угол падения. Тогда для средней по реализациям шероховатой поверхности интенсивности отраженного поля $I=\left\langle|u|^{2}\right\rangle[6]$, путем подстановки поля в виде (4) и вычисления пространственных интегралов [12] получается выражение

$$
I=\frac{k_{z}^{2} S}{2 \pi^{3} R_{1}^{2}} F\left(Q_{x}, K\right) \delta\left(Q_{y}\right) .
$$

Здесь множитель

$$
\left.F\left(Q_{x}, K\right)=\int\langle\exp \{i K \mid \xi(x)-\xi(0)]\}\right\rangle \exp \left(i Q_{x} x\right) d x,
$$

$\langle\exp \{i K[\xi(x)-\xi(0)]\}\rangle$ есть среднее по ансамблю поверхностей значение фазового множителя, а $S$ - площадь отражающей поверхности. При выводе уравнений (5) и (6) предполагалось, что длина полосы намного больше как длины волны, так и ширины полосы. Наличие множителя $\delta\left(Q_{y}\right)$ в уравнении (6) фиксирует условие зеркального отражения вдоль оси $y$, которое выполняется в пределе больших длин полос. При конечной протяженности полосы, с которой фактически приходится иметь дело, этот множитель имеет вид дифракционного фактора с конечной угловой шириной, которая, однако, не зависит от шероховатости поверхности и значительно меньше угловой ширины рассеяния в поперечном направлении, а поэтому не представляет интереса в данной задаче. Далее мы ограничимся случаем, когда $Q_{y}=0$, а $Q_{x}=Q$.

При выполнении условия $K \xi \ll 1$ часть амплитуды поля рассеяния, обусловленная неоднородностями, определяется фактором

$$
F(Q, K)-1=K^{2} \int\langle\xi(x) \xi(0)\rangle \exp (i Q x) d x,
$$

т.е. выражается через спектр корреляционной функции $\langle\xi(x) \xi(0)\rangle$ высот шероховатой поверхности. Это соответствует стандартному результату применения метода малых возмущений Релея [7]. Важно отметить, что модель фазового экрана обеспечивает правильный вид предельного выражения в области, где исходное приближение ФО формально не является справедливым, поскольку условием его выполнимости является требование, чтобы размеры рассеивающей неоднородности были больше длины волны. Это делает возможным использовать уравнения (5) и (6) для параметризации рассеяния, как на крупных, так и на мелких шероховатостях. При больших шероховатостях приближение корреляционной функции высот становится недостаточным, так что для вычисления интеграла в уравнении (6) приходится применять разложение по кумулянтам [31]. Для гауссова процесса конечный результат можно выразить через корреляционную функцию [27].

\section{Модель диффузии фазы}

Уравнение (6) совпадает по виду с корреляционной функцией гармонических колебаний со случайны- 
ми фазами, для которой соответствующая теория развита в статистической радиофизике [32]. Вычисление среднего по ансамблю $\exp \{i K[\xi(x)-\xi(0)]\}$ возможно при задании конкретного распределения величины $\chi=\xi(x)-\xi(0)$, например, в предположении, что это нормальный процесс диффузионного типа с функцией распределения

$$
p(\chi)=\frac{1}{\sqrt{2 \pi f}} \exp \left(-\frac{\chi^{2}}{2 f}\right),
$$

где $f(x)=\left\langle[\xi(x)-\xi(0)]^{2}\right\rangle$. В этом случае прямое вычисление дает

$$
\langle\exp \{i K[\zeta(x)-\xi(0)]\}\rangle=e^{-f K^{2} / 2}
$$

Дальнейшее продвижение в определении дисперсии $f(x)$ может быть основано на дополнительной информации о характере самого случайного процесса $\xi(x)$. Одним из простейших является представление о том, что девиация шероховатости в виде $\Omega(x)=d \xi(x) / d x$ представляет собой стационарный случайный процесс, для которого $\langle\Omega(x)\rangle=0$, а функция корреляции

$$
\langle\Omega(x) \Omega(0)\rangle=\int_{0}^{\infty} g_{\Omega}(\kappa) e^{-i \kappa x} d \kappa
$$

где $g_{\Omega}(\kappa)$ - ее спектральная плотность. Для случайного накопления величины $\xi$ вдоль пути от 0 до $x$ имеем

$$
\zeta(x)-\zeta(0)=\int_{0}^{x} \Omega(x) d x
$$

Для среднего квадрата $\xi(x)-\xi(0)$ можно получить [33] соотношение

$$
\left\langle[\zeta(x)-\zeta(0)]^{2}\right\rangle=2 \int_{0}^{\infty} g_{\Omega}(\kappa) \frac{1-\cos \kappa x}{\kappa^{2}} d \kappa .
$$

Таким образом, среднеквадратичный набег высоты полностью определяется спектром случайной девиации волнового числа $\Omega(x)$.

Исходя из уравнения (8), определим закон ,диффузии“ $f(x)$ для двух асимптотических частных случаев распределения шероховатостей по масштабам, когда их спектр $g_{\Omega}(\kappa)$ широкий и когда он узкий. Для характеристики спектра введем средний квадрат флуктуаций

$$
\overline{\Omega^{2}}=\int_{0}^{\infty} g_{\Omega}(\kappa) d x
$$

Относительная протяженность спектра задается значением параметра $\eta=\overline{\Omega^{2}} K^{2} l^{2}$, где $l$ - длина корреляции. Величина $l$ определяется тем, что функция $K^{2} g_{\Omega}(\kappa)$ заметно отлична от нуля только при $\kappa l<1$.
Рассмотрим вначале случай, когда

$$
\eta \gg 1 \text {. }
$$

Условие (9) означает, что „высота“ $\overline{\Omega^{2}} K^{2} l$ спектра гораздо больше его ширины $1 / l$, и спектр $K^{2} g_{\Omega}(\kappa)$ узкий. Тем самым шероховатости сильно изменяются по величине на больших масштабах, т.е. являются крупными. Для узкого спектра в подынтегральном выражении уравнения (8) можно положить $1-\cos \kappa x \approx \kappa^{2} x^{2} / 2$, и

$$
f(x)=x^{2} \int_{0}^{\infty} g_{\Omega}(\kappa) d \kappa=\overline{\Omega^{2}} x^{2} .
$$

Согласно уравнениям (6), (7) и с учетом (10), имеем

$$
F(Q)=\int \exp \left(-K^{2} \overline{\Omega^{2}} x^{2} / 2\right) \exp (i Q x) d x .
$$

Экспонента обрезает подынтегральное выражение на расстояниях $x^{2} \sim 1 / K^{2} \overline{\Omega^{2}} \ll l^{2}$. В результате интегрирования будем иметь

$$
F(Q)=\frac{1}{2 k \cos \theta \sqrt{2 \pi \Omega^{2}}} \exp \left(-\frac{\operatorname{tg}^{2} \theta}{2 \Omega^{2}}\right),
$$

т.е. получается характерная форма спектрального фактора рассеяния для крупномасштабной плавной шероховатой поверхности [24,34].

Второй предельный случай возникает, когда $\eta \ll 1$, и изменения шероховатости поверхности характеризуются малыми по величине масштабами, а спектр $K^{2} g_{\Omega}(\kappa)$ является широким. В этом случае мы имеем дело с мелкими шероховатостями, а функцию $g_{\Omega}(\kappa)$ в подынтегральном выражении уравнения (8) можно считать плавной и вынести за знак интеграла. Оставшийся интеграл вычисляется [35], и результат имеет вид

$$
f(x)=2 g_{\Omega}(0) \int_{0}^{\infty} \frac{1-\cos \kappa x}{\kappa^{2}} d \kappa=\pi g_{\Omega}(0)|x| .
$$

С учетом уравнения (7) теперь получаем

$$
F(Q)=\frac{2 \pi g_{\Omega}(0)}{\pi^{2} g_{\Omega}(0)^{2} k^{2} \cos ^{2} \theta+\operatorname{tg}^{2} \theta},
$$

и фактор рассеяния приобретает форму лоренцева типа, когда убывание с ростом угла падения происходит медленнее экспоненциального, имеющего место, согласно уравнению (11), для крупных неоднородностей. На рис. 2 показана зависимость относительной величины интенсивности обратного рассеяния от угла падения $\theta$ на подстилающую плоскость для крупных и мелких шероховатостей, нормированная на единицу в максимуме при $\pi g_{\Omega} k=\sqrt{\overline{\Omega^{2}}}$. Как для широкого, так и для узкого спектра шероховатостей максимальная интенсивность рассеяния достигается при $Q=0$, т.е. при 


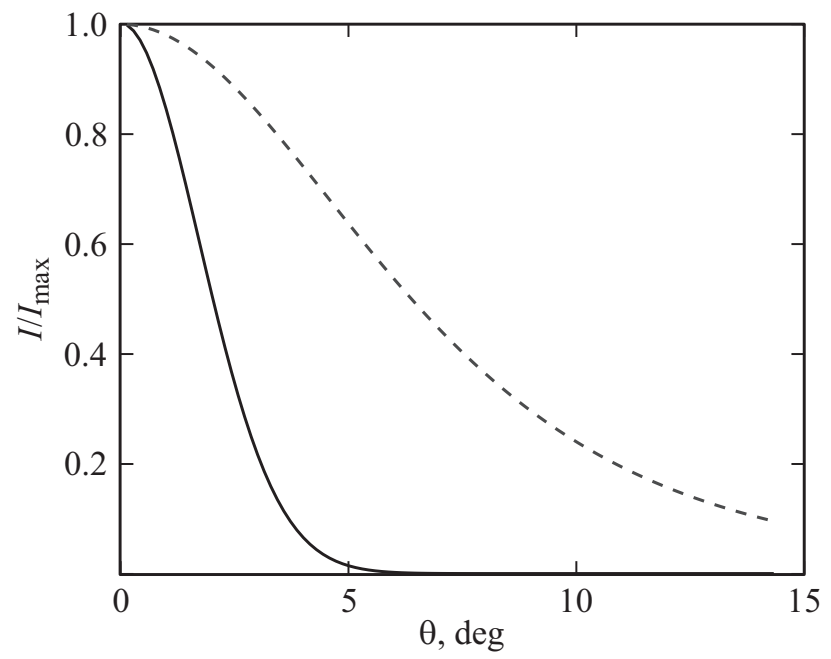

Рис. 2. Зависимость интенсивности рассеянной назад волны от угла падения: сплошная линия - рассеяние на крупных шероховатостях $(\eta \gg 1)$; штриховая линия - рассеяние на мелких шероховатостях $(\eta \ll 1)$.

нормальном падении. Вывод о характере зависимости обратного рассеяния от угла падения при разной ширине спектра девиации флуктуаций шероховатостей носит общий характер и подтверждается результатами прямого численного моделирования [36].

\section{Метод фазового скачка}

Рассеяние волн неровной поверхностью критическим образом зависит от статистики шероховатостей [37-39]. Для сложной статистики расчет рассеяния с учетом фазовой модуляции отраженного поля можно проводить путем прямого численного моделирования, вычисляя интеграл в уравнении (6), а затем усредняя по ансамблю реализаций шероховатой поверхности. Для ступенчатой зеркальной поверхности интеграл в уравнении (6) представляется в виде суммы по участкам из отдельных гладких полос постоянной высоты $\xi_{m}$ и ширины $l_{m}$, так что структурный фактор

$$
F(Q, K)=\sum_{m} e^{i \varphi_{m}} \frac{\sin \left(Q l_{m} / 2\right)}{Q / 2},
$$

где фаза

$$
\varphi_{m}=K \xi_{m}+Q\left(\sum_{k=1}^{m} l_{j}-l_{m} / 2\right) .
$$

В результате исходная плоская гармоническая волна после отражения будет представлена в дальней зоне суммой волн с различной амплитудой и фазой, т.е. в виде сигнала с амплитудно-фазовой модуляцией. Фаза отдельной компоненты суперпозиции волн складывается из текущей модуляции за счет высоты ступеньки $\zeta_{m}$ и набега фазы при наклонном падении на длине $\sum_{k=1}^{m} l_{j}-l_{m} / 2$ от края поверхности до середины текущей полосы с номером $m$. Она меняет свое значение дискретно при переходе к следующей полосе, что отражает сущность метода фазового скачка $[25,26]$. Амплитуда отдельной компоненты определяется множителем, который задает острую направленность рассеянной волны под углом отражения при больших значениях $l_{m}$, когда $k l_{m} \gg 1$. В обратном пределе, когда $k l_{m} \ll 1$, рассеяние волны отдельной ступенькой теряет свою направленность, поскольку $\sin \left(Q l_{m} / 2\right) /(Q / 2) \approx l_{m}$. Таким образом, первый сомножитель в суммируемом выражении уравнения (13) отвечает за когерентность сложения волн, а второй за амплитуду и направленность отдельной компоненты, обусловленной дифракцией на соответствующей полосе. При $Q \neq 0$ прогрессирующее накопление фазы происходит случайными скачками, что подкрепляет использованную выше модель диффузии фазы.

Расширение рассматриваемой схемы с поворотом горизонтальных отражающих полос на разные углы вокруг их продольной оси приведет к одномерной модификации метода касательных отражающих плоскостей [23,24]. Пусть шероховатость в виде зеркальных ступенек распо-
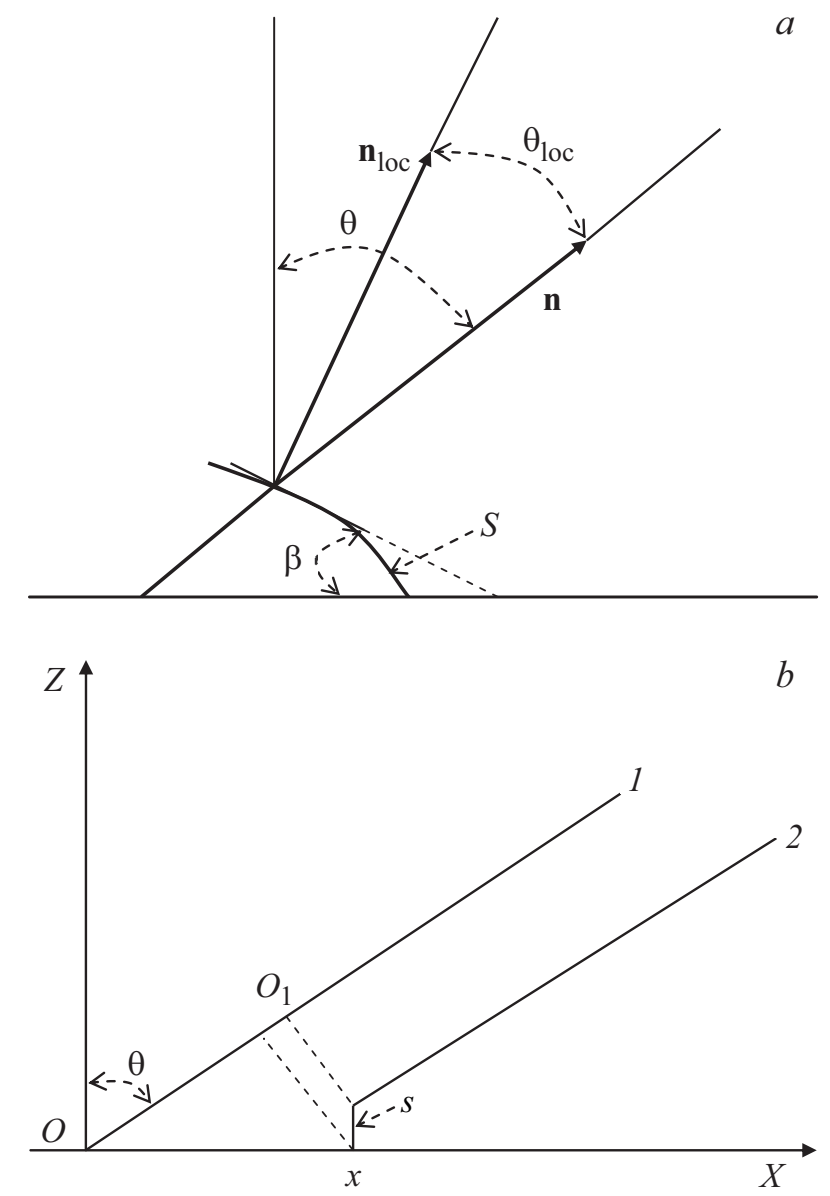

Рис. 3. Геометрия рассеяния и обозначения для криволинейной поверхности: $(a)$ взаимное расположение локальной нормали к поверхности $\mathbf{n}_{\text {loc }}$ и направления рассеяния $\mathbf{n}$; $(b)$ разность хода $O O_{1}$ между лучами 1 и 2 , идущими от разных точек поверхности. 
лагается на плавно изменяющейся по высоте подстилающей поверхности $s(x)$. Геометрия гладкой составляющей поверхности и обозначения показаны на рис. $3, a, b$. Основания рассеивающих элементов представляют собой теперь касательные к кривой $s(x)$ отрезки длиной $l_{j}$. Проекция конца такого элемента $m$ на горизонтальную ось

$$
x_{m}=\sum_{j=1}^{m} \frac{l_{j}}{\sqrt{1+\dot{s}_{j}^{2}(x)}},
$$

а для локального угла падения на поверхность

$$
\cos \theta_{\mathrm{loc}}=\frac{\cos \theta+\dot{s}(x) \sin \theta}{\sqrt{1+\dot{s}^{2}(x)}} .
$$

Изменение фазы отраженной волны с учетом ее набега вдоль криволинейной поверхности в соответствии с рис. $3, b$ составит

$$
\varphi_{m}=2 k\left[\xi_{m} \cos \theta_{m}+s\left(x_{m-1 / 2}\right) \cos \theta+x_{m-1 / 2} \sin \theta\right],
$$

а структурный фактор примет вид

$$
F(Q, K)=\sum_{m} e^{i \varphi_{m}} \frac{\sin \left(Q_{m} I_{m} / 2\right)}{Q_{m} / 2},
$$

где $Q_{m}=2 k \sin \theta_{\text {locm. }}$ Половинный индекс в формуле (14) указывает, что значение координаты взято в середине интервала с номером $m$.

При численных расчетах требуется следить за представительностью выборки и достигаемой точностью вычислений во избежание артефактов, к которым, по всей видимости, относятся мелкие осцилляции угловой зависимости в ранних работах $[40,41]$, обусловленные недостаточными для сглаживания результата размерами сетки дискретизации и малым числом элементов в ансамбле реализаций, по которым проводилось усреднение. Более поздние результаты [39] таких нерегулярных отклонений уже не содержат.

\section{Комбинированные поверхности и результаты численных расчетов}

Для численного расчета интенсивности воспользуемся ее состоятельной оценкой [42]

$$
\bar{I}=\frac{k_{z}^{2}}{R_{1}^{2}} \frac{1}{n} \sum_{j=1}^{n} \frac{1}{X}\left|\int_{(j-1) X}^{j X} \exp [i Q x+i K \zeta(x)] d x\right|^{2},
$$

где длина каждого из $n$ интервалов интегрирования $X \gg l$. Оценка (15) обеспечивает стремление к нулю дисперсии при $X \rightarrow \infty, n \rightarrow \infty$. Конечность числа интервалов приводит к неполному статистическому усреднению по возможным реализациям, а конечность длины интервала $X$, в случае сопоставимости области рассеяния волны с масштабом корреляций шероховатостей, частично сохраняет когерентность рассеянных волн.

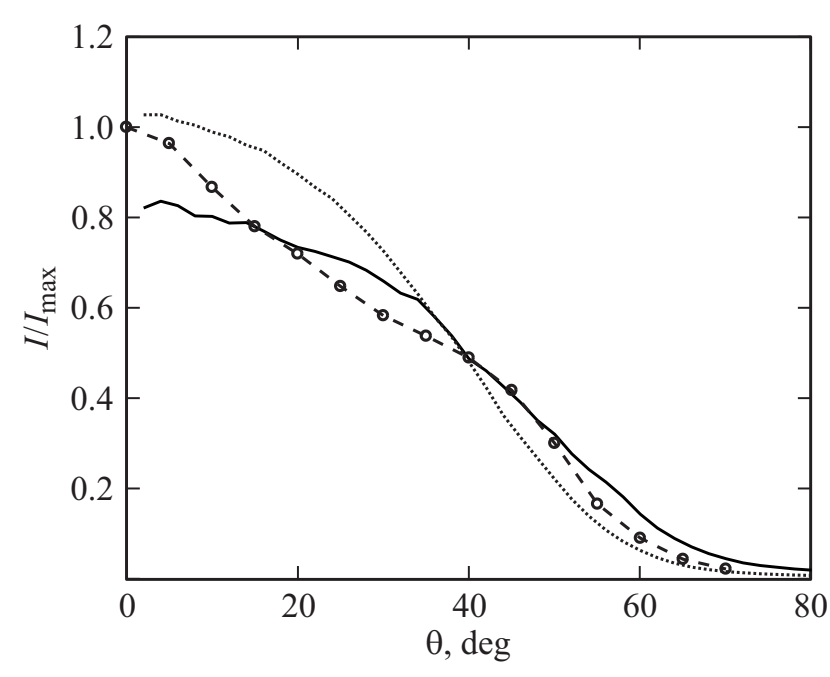

Рис. 4. Зависимость от угла падения интенсивности обратного рассеяния на синусоидальной шероховатой поверхности: сплошная линия - результаты расчета методом фазового скачка, пунктирная - результат расчета методом касательных отражающих плоскостей, точки, соединенные штриховой линией - экспериментальные данные [16].

В качестве примера применения метода фазового скачка рассмотрим рассеяние на крупномасштабной периодической решетке, модулированной мелкомасштабными случайными неоднородностями, так что локальная средняя высота меняется по закону

$$
s(x)=A \sin (2 \pi x / L)
$$

с периодом $L$ и амплитудой $A$, а случайные неоднородности на гладкой поверхности описываются гауссовым процессом $\xi(x)$ с корреляционной функцией

$$
\langle\xi(x) \xi(0)\rangle=a^{2} \exp \left(-x^{2} / 2 l^{2}\right),
$$

при стандартном отклонении $a$ высоты шероховатости $\xi$ и корреляционной длине $l$. Численный алгоритм генерации случайной функции с заданным распределением значений и определенной длиной корреляции описан в [43]. Гауссова функция распределения наклонов, соответствующая корреляционной функции (16), есть

$$
P_{G}\left(\zeta_{x}\right)=\frac{1}{\sqrt{2 \pi} a} \exp \left(-\frac{\xi_{x}^{2} l^{2}}{2 a^{2}}\right) .
$$

На рис. 4 показан результат расчета зависимости относительной величины интенсивности от угла падения для обратного рассеяния микроволнового излучения с длиной волны $\lambda=0.6328 \mu \mathrm{m}$ на зеркальной шероховатой поверхности с $A=10 \mu \mathrm{m}, L=1 \mathrm{~mm}, a=0.56 \mu \mathrm{m}$ и $l=2.17 \mu \mathrm{m}$ с учетом случайных вариаций локального наклона поверхности за счет шероховатостей. При численном моделировании число элементарных отражающих полос составляло $\sim 10^{3}$, а ансамбль для 
усреднения включал $3 \cdot 10^{3}$ элементов. Расчеты проводились методом фазового скачка и методом касательных отражающих плоскостей, в котором когерентный учет сложения отраженных волн проводился в пределах каждой зеркальной полосы, а интенсивности от разных полос складывались. Выбранные расчетные параметры соответствовали условиям экспериментальных измерений [16] углового распределения света, рассеянного поверхностью, состоящей из мелкой шероховатости, наложенной на медленно изменяющуюся поверхность. Крупномасштабные поверхности представляли собой обработанные алмазом периодические синусоидальные профили, изготовленные из алюминия. Методом микролитографии на поверхность была добавлена мелкая шероховатость из золота с коэффициентом отражения волн $s$-поляризации 0.95. Сравнение результатов расчетов, нормированных на максимальное экспериментальное значение в нуле, с измеренной угловой зависимостью полного рассеяния волн $s$-поляризации хорошо отражающей поверхностью золота (без учета поляризации рассеянного излучения) показывает, что метод фазового скачка дает значения более близкие к данным измерений. В то же время полного совпадения не наблюдается, что, по всей видимости, указывает на необходимость дополнительного учета поляризационных эффектов в рамках векторной теории.

В более сложном примере выбранная нами рассеивающая поверхность описывается основным мелкомасштабным случайным процессом, который дополнительно модулируется случайным образом от большой величины к малой и обратно на значительных масштабах, задавая аппликативную поверхность. Уточним статистическую модель аппликативной поверхности. Подходящий для этого тип случайного процесса известен в радиофизике как случайный сигнал с замиранием [44]. Математически эффект замирания представляется мультипликативным образом в виде

$$
h(x)=s(x) \xi(x)
$$

где $s(x)$ означает медленно изменяющуюся с расстоянием случайную величину, а $\zeta(x)$ определяет мелкие случайные изменения локального рельефа.

Основной случайный процесс $\xi(x)$ определяет шероховатость поверхности при отсутствии медленной модуляции. Мы зададим его с помощью одностороннего распределения Релея [45]

$$
\begin{aligned}
& P_{R}(u, a)=\frac{u}{a^{2}} \exp \left(-\frac{u^{2}}{2 a^{2}}\right), \\
& \langle u\rangle=(\pi / 2)^{1 / 2} a, \quad\left\langle u^{2}\right\rangle=2 a^{2} .
\end{aligned}
$$

Выбор в пользу распределения Релея [46] обусловлен потребностью описания строго положительных величин, что невозможно с помощью распределения Гаусса, не ограниченного на числовой оси. Протяженности ступенек $l$ зададим распределением $P_{R}\left(l, a_{l}\right)$, а независимое распределение их высот $\xi-P_{R}\left(\xi, a_{\xi}\right)$. Ступеньки

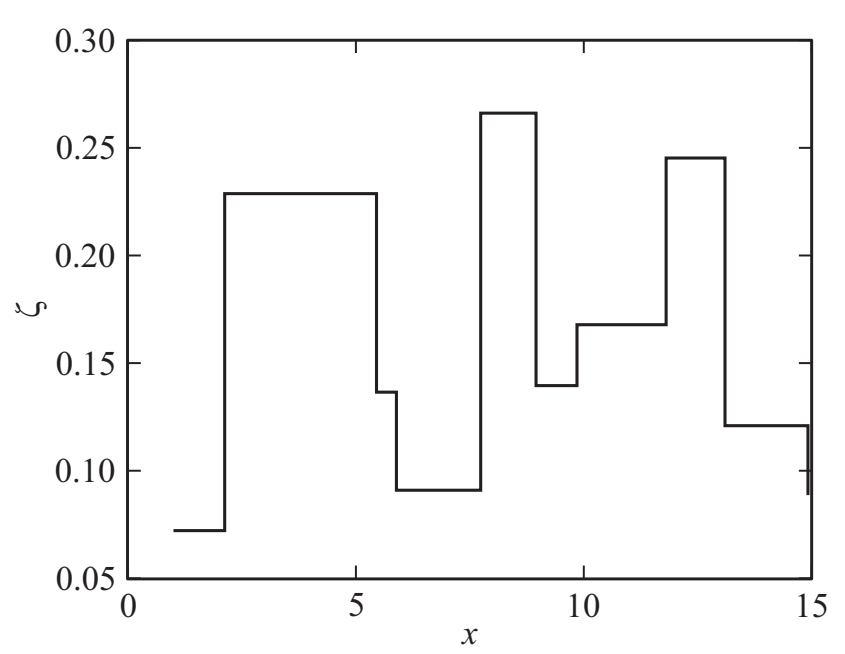

Рис. 5. График профиля случайной ступенчатой поверхности со статистикой Релея.

примыкают друг к другу, и $a_{l} \gg a_{\xi}$. Это позволяет не учитывать перерассеяние поля волны без явного ограничения высоты рельефа. Эффект замирания можно получить, задав дополнительно распределения Релея $P_{R}\left(\beta, a_{\beta}\right)$ для коэффициента модуляции рельефа $\beta$ $(\xi \rightarrow \beta \xi)$ и распределение $P_{R}\left(L, a_{L}\right)$ для интервалов $L$ модулирующего медленного процесса, с условием $a_{L} \gg a_{l}$.

На рис. 5 показан пример графика случайной ступенчатой функции $\zeta(x)$ со ступеньками разной протяженности и высоты при фиксированном среднем отношении ширины к высоте, равном 10. Для такого профиля поверхности проведен расчет углового распределения обратного рассеяния при $\lambda=a_{l} / 10$ с усреднением зависимости по ансамблю реализаций. Результаты расчетов представлены на рис. 6. Они показывают наличие резкого пика при малых углах, демонстрирующего тот факт, что дифракционное уширение углового спектра на широких плоских ступеньках в среднем мало. Аналогичные расчеты для $\lambda=a_{l} / 2$ отчетливо выявляют тенденцию уширения углового спектра за счет роста дифракционной расходимости волн, отраженных от отдельных участков поверхности, в согласии с оценкой $\sim 3^{\circ}$ для этого случая. Такое характерное поведение еще нагляднее проявляется при $\lambda=2.2 a_{l}$. Повторная, но крупномасштабная (мультипликативная) случайная модуляция поверхности приводит к появлению в зависимости особенности, заключающаяся в наличии острого максимума при малых углах наблюдения и полого убывающей интенсивности при больших углах. Эта двойственность объясняется тем, что в распределении заметно представлены протяженные участки с высотами неоднородностей как больше, так и намного меньше длины волны. Для сравнительно гладких участков проявляется дифракционное усиление в направлении $\theta \approx 0$, в то время как участки с большей шероховатостью вызывают диффузное рассеяние. Этот факт подтверждает общий 


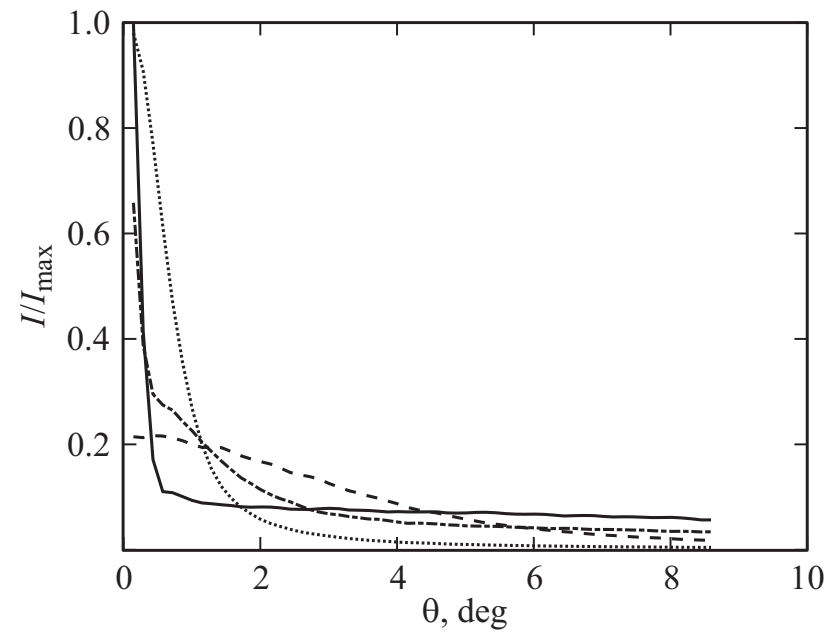

Рис. 6. Усредненная по реализациям случайной поверхности зависимость интенсивности обратного рассеяния от угла: $\lambda=a_{l} / 10$ (сплошная линия), $\lambda=a_{l} / 2$ (пунктир); $\lambda=2.2 a_{l}$ (штриховая линия), результат расчета при дополнительной случайной крупномасштабной модуляции шероховатости, $a_{L}=10 a_{l}$ (штрихпунктир).

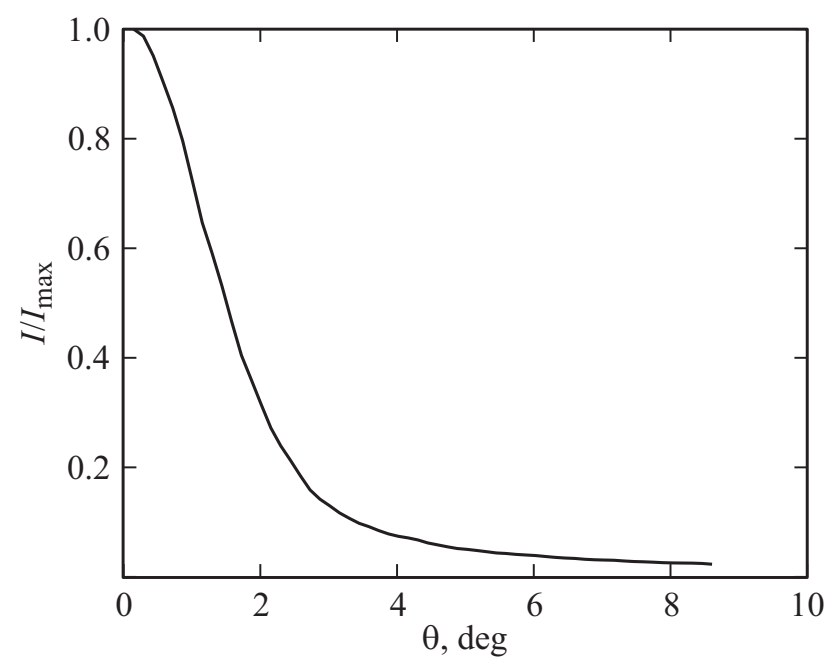

Рис. 7. Рассеяние назад аддитивной двухмасштабной поверхностью.

качественный вывод, сделанный выше на основе асимптотических аналитических оценок, о том, что крупномасштабные неоднородности порождают экспоненциально спадающий спектр, а мелкомасштабные неоднородности дают плавное лоренцево убывание интенсивности. В модели фазового скачка излом в зависимости возникает естественным образом из-за разных условий дифракции на сглаженных и рифленых участках.

На рис. 7 для сравнения представлен результат расчета зависимости относительной величины интенсивности обратного рассеяния от угла падения для двухмасштабной шероховатой поверхности при аддитивном характеpe неровностей с теми же параметрами, что и выше в мультипликативной модели при $\lambda=2.2 a_{l}$. Он ожидаемо демонстрирует сужение углового распределения, хорошо известное в двухмасштабной аддитивной модели, но существенно менее выраженное, чем в мультипликативном варианте. Так проявляется роль крупномасштабной подстилающей поверхности, создающей более однородный фон, на котором все мелкомасштабные флуктуации рельефа формируют диффузное рассеяние.

\section{Заключение}

Рассмотренная в работе скалярная модель рассеяния волн шероховатой поверхностью в виде фазового экрана является основой для расчета обратного рассеяния света от хорошо отражающих поверхностей с различной статистикой неровностей. Реализация модели фазового экрана в методе фазового скачка дает необходимый численный инструмент. Он обеспечивает возможность расчета рассеяния излучения случайной одномерной ступенчатой зеркальной поверхностью при разных статистических параметрах шероховатостей, меняющих итоговую диаграмму обратного рассеяния. Для аппликативной поверхности, в которой чередуются участки с разными характерными амплитудами шероховатостей, продемонстрирован эффект увеличения рассеянного назад излучения в области малых углов падения. Отметим, что использованное скалярное приближение Кирхгофа не описывает зависимость рассеяния от поляризации для реальных отражающих поверхностей. Если поверхность не является идеальным зеркалом, метод следует модифицировать с применением формул Френеля для коэффициентов отражения или импедансного граничного условия Леонтовича [9]. В общем случае коэффициент отражения неровной поверхностью зависит от поляризации волны, меняясь от точки к точке поверхности в зависимости от угла падения волны. Дальнейшее развитие метода фазового скачка в применении к задаче рассеяния электромагнитных волн шероховатыми поверхностями предполагает учет влияния поляризации на рассеяние, исходя из векторного варианта уравнения Кирхгофа-Гельмгольца, а также поиск равномерных по длине волны асимптотик рассеяния элементарными поверхностями, которые позволят точнее описывать обратное рассеяние при произвольной статистике неровностей.

\section{Конфликт интересов}

Авторы заявляют, что у них нет конфликта интересов.

\section{Список литературы}

[1] Хусу А.П., Виттенберг Ю.Р., Пальмов В.А. Шероховатость поверхностей (теоретико-вероятностный подход). М.: Наука, 1975. 343 с.

[2] Карасик В.Е., Орлов В.М. Локационные лазерные системы видения. М.: Изд-во МГТУ им. Н. Э. Баумана, 2013. $478 \mathrm{c}$. 
[3] Основы импульсной лазерной локации / Под ред. В.Н. Рождествина. М.: Изд-во МГТУ, 2010. 573 с.

[4] Волосюк В.К., Кравченко В.Ф. Статистическая теория радиотехнических систем дистанционного зондирования в радиолокации. М.: ФИЗМАТЛИТ, 2008. 704 с.

[5] Локшин Г.Р. Основы радиооптики. Долгопрудный: Издательский дом „Интеллект“, 2014. 344 с.

[6] Рытов С.М., Кравцов Ю.А., Татарский В.И. Введение в статистическую радиофизику. Случайные поля. Ч. 2. М.: Наука, 1978. 463 с.

[7] Elfouhaily T.M., Guérin C.-A. // Waves in Random Media. 2004. V. 14. P. R1-R40.

[8] Voronovich A.G. Wave Scattering from Rough Surface. Berlin: Springer, 1999. $236 \mathrm{p}$.

[9] Фейнберг Е.Л. Распространение радиоволн вдоль земной поверхности. М.: Наука, 1999. 496 с.

[10] Стретт Джс.В. Теория звука. Т. II. М.: ГИТТЛ, 1955. C. 94.

[11] Beckmann P., Spizzichino A. The Scattering of Electromagnetic Waves from Rough Surfaces. Norwood: Artech House, 1987. 511 p.

[12] Басс Ф.Г., Фукс И.М. Рассеяние волн на статистически неровной поверхности. М.: Наука, 1972. 424 с.

[13] Nieto-Vesperinas M., García N. // Opt. Acta. 1981. V. 28. P. $1651-1672$.

[14] Light Scattering and Nanoscale Surface Roughness / Ed. by A.A. Maradudin. N.Y.: Springer, 2007. 496 p.

[15] Курьянов Б.Ф. // Акустический журнал. 1962. Т. 8. C. $325-333$.

[16] Ruiz-Cortés V., Dainty C. // J. Opt. Soc. Am. A. 2012. V. 29. N 6. P. 1154-1160.

[17] Boukabara S.A., Eymard L., Guillou C., Lemaire D., Sobieski P., Guissard A. // Radio Science. 2002. V. 37. P. $1-11$.

[18] Lemaire D., Sobieski P., Craeye C., Guissard A. // Radio Science. 2002. V. 37. P. 1001(16).

[19] Nunziata F., Sobieski P., Migliaccio M. // EEE Trans. on Geoscience and Remote Sensing. 2009. V. 47. P. 1949-1956.

[20] Mouche A.A., Chapron B., Reul N. // Waves in Random and Complex Media. 2007. V. 17. P. 321-341.

[21] Головинский П.А., Проскурин Д.К. // Научно-технические ведомости СПбГПУ. Физико-математические науки. 2018. T. 11. № 4. C. 112-120. doi 18721/JPM.11411

[22] Ayari M.Y., Khenchaf A., Coatanhay A. // J. Appl. Remote Sensing. 2007. V. 1. P. 013532(19).

[23] Ishimaru A. Electromagnetic Wave Propagation, Radiation and Scattering. Hoboken: John Wiley \& Sons, 2017. 944 p.

[24] Brekhovskikh L.M., Lysanov Yu.P. Fundamentals of Ocean Acoustics. Berlin: Springer-Verlag, 1991. 270 p.

[25] Дифракционная оптика и нанофотоника / Под ред. Сойфеpa В.А. М.: ФИЗМАТЛИТ, 2014. 608 с.

[26] Khonina S.N., Balalaev S.A., Skidanov R.V. et al. // J. Opt. A: Pure Appl. Opt. 2009. V. 11. P. 065702-065708.

[27] Рогаткин А.Д. // Опт. и спектр. 2004. Т. 97. В. 3. C. $484-493$.

[28] Уфимцев П.Я. Основы физической теории дифракции. М.: БИНОМ. Лаборатория знаний, 2014.

[29] Залипаев В.В., Костин А.В. // ЖТФ. 2000. Т. 70. В. 1. C. 3-9.

[30] Osipov A.V., Tretyakov S.A. Modern Electromagnetic Scattering Theory with Applications. Chichester: Wiley \& Sons, 2017. 806 p.
[31] Winebrenner D.P., Ishimaru A. // J. Opt. Soc. Am. A. 1985. V. 2. N 12. P. 2285-2294.

[32] Стратонович Р.Л. Случайные процессы в динамических системах. М.; Ижевск: Институт компьютерных исследований, НИЦ „Регулярная и хаотическая динамика“, 2009. $502 \mathrm{c}$.

[33] Рытов С.М. Введение в статистическую радиофизику. Случайные процессы. Ч. 1. М.: Наука, 1976. 495 с.

[34] Brown G.S. // IEEE Trans. on Antennas and Propagation. 1978. V. AP-26. N 3. P. 472-482.

[35] Градштейн И.С., Рыжик И.М. Таблицы интегралов, рядов и произведений. СПб.: БХВ-Петербург, 2011. 1232 с.

[36] González-Alcalde A.K., Méndez E.R., Terán E., Cuppo F.L.S., Olivares J.A., Garsi-Valenzuela A. // J. Opt. Soc. Am. A. 2016. V. 33. P. 373-382.

[37] Jakeman E., Ridley K.D. Modeling Fluctuations in Scattered Waves. NY.: Taylor and Francis Group, 2006. 336 p.

[38] Bruce N.C. // Appl. Opt. 2005. V. 44. P. 784-791.

[39] Simonsen I., Hetland Ø.S., Kryvi J.B., Maradudin A.A. // Phys. Rev. A. 2016. V. 93. P. 043829(15).

[40] Soto-Crespo J.M., Nieto-Vesperinas M. // J. Opt. Soc. Am. A. 1989. V. 6. N 3. P. $387-384$.

[41] Michel T., Maradudin A.A., Méndez E.R. // J. Opt. Soc. Am. B. 1989. V. 6. N 12. P. 2438-2446.

[42] Свешников А.А. Прикладные методы теории случайных функций. СПб.: Изд-во „Лань“, 2011. 464 с.

[43] Fung A.K., Chen M.F. // J. Opt. Soc. Am. A. 1985. V. 2. N 12. P. 2274-2284.

[44] Jain S., Barona C., Madamopoulos N. // J. Cyber Security. 2016. V. 4. P. 279-304.

[45] Ajami M., Jahanshahi S.M.A. // J. Mod. Appl. Statistical Methods. 2017. V. 16. N 2. P. 256-276.

[46] Zhao Y.-P., Wang G.-C., Lu T.-M. // Phys. Rev. B. 1997. V. 55. N 20. P. 13938-13952. 